\title{
The incidence of silicone oil-related visual loss following the removal of heavy silicone oil
}

\author{
J. Y. X. Lee ${ }^{1} \cdot$ R. Sawant ${ }^{1} \cdot$ A. Jonas ${ }^{2} \cdot$ J. Lochhead ${ }^{1}$ \\ Received: 29 January 2019 / Revised: 20 April 2019 / Accepted: 16 May 2019 / Published online: 2 July 2019 \\ (c) The Royal College of Ophthalmologists 2019
}

\section{Introduction}

Unexplained visual loss following the removal of silicone oil (ROSO) has been termed silicone oil-related visual loss or silicone oil maculopathy $[1,2]$. The incidence has been estimated to be $\sim 3.3-5.9 \%$. The British Ophthalmological Surveillance Unit are currently collecting data from the United Kingdom with reference to these cases, which are defined as unexplained loss following ROSO of two or more lines of Snellen visual acuity (VA) or deterioration to worse than 6/60 from best corrected VA (BCVA) with SO in situ, in patients with macula on retinal detachments (RD). No cases have previously been reported in relation to heavy SO (HSO) and we therefore conducted a review of HSO cases to determine the incidence of these events.

\section{Methods}

We performed a retrospective review of cases undergoing removal of HSO (ROHSO) at our institution from 2006 to 2018. These cases were further sub-classified into two distinct groups: HSO (Densiron 68) alone and mixed HSO (Densiron 68 combined with conventional 1300cs SO). The ROHSO was performed using 23- or 25-gauge pars plana vitrectomy (PPV) with an additional 18-gauge port for active aspiration down to the optic disc.

Data for the BCVA was collected 2-3 months following ROHSO and compared with the BCVA pre-operatively.

J. Y. X. Lee

jayne.lee@doctors.org.uk

1 Ophthalmology Department, St. Mary's Hospital, Isle of Wight NHS Trust, Parkhurst Road, Isle of Wight PO30 5TG, UK

2 Southampton General Hospital, University Hospital Southampton NHS Foundation Trust, Southampton, UK
Patients with any unexplained loss of two or more lines of Snellen acuity were then identified and investigated for signs of silicone oil maculopathy.

\section{Results}

We identified 41 eyes which underwent ROHSO. Thirty-two eyes underwent removal of HSO alone and nine eyes had removal of mixed HSO.

Two cases $(4.9 \%)$ were identified with unexplained visual loss following the ROHSO: one in the HSO alone group $(3.1 \%)$ and one in the mixed HSO group $(11.1 \%)$. Both patients were treated for macula on RD with BCVA of $6 / 9$ before ROHSO. In both cases, the visual loss was greater than three lines of Snellen VA and occurred immediately following ROHSO with no documented recovery during the follow up period.

Both patients demonstrated a relative central scotoma following ROHSO with initially unremarkable macula optical coherence tomography (OCT) appearances, as shown in Fig. 1a, b taken 2 months after ROHSO. Diffuse, fine scattered microcysts became evident later as previously described in other reports [3].

\section{Discussion}

Our study is limited in numbers but suggests an overall comparable incidence of $4.9 \%$ following ROHSO.

This phenomenon remains unexplained but there have been several hypotheses proposed, including phototoxicity from the operating microscope and the refractive behaviour of a shrinking SO bubble inside the eye [4]. This is unlikely to have contributed in our cases as the entire ROHSO procedure was performed with endoillumination. In addition, ROHSO involves the oil bubble shrinking down towards the optic disc instead of collecting behind the lens. Sudden changes in potassium ion concentration 
a

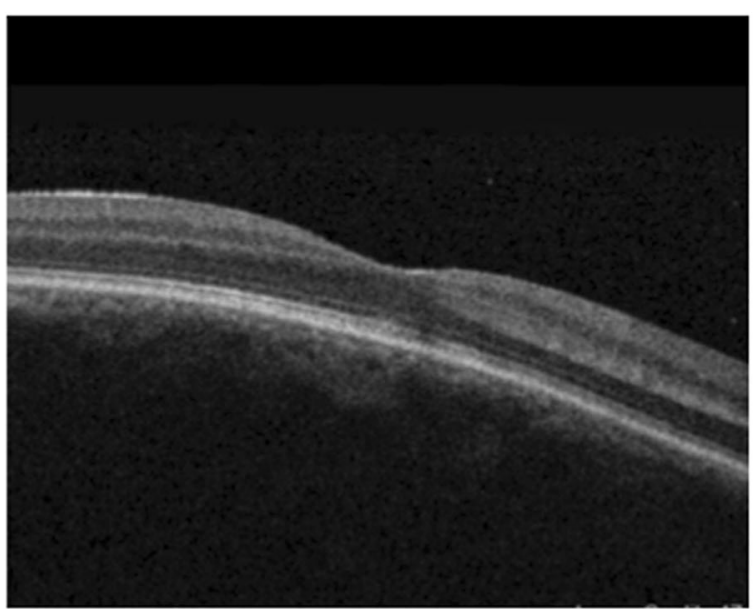

b

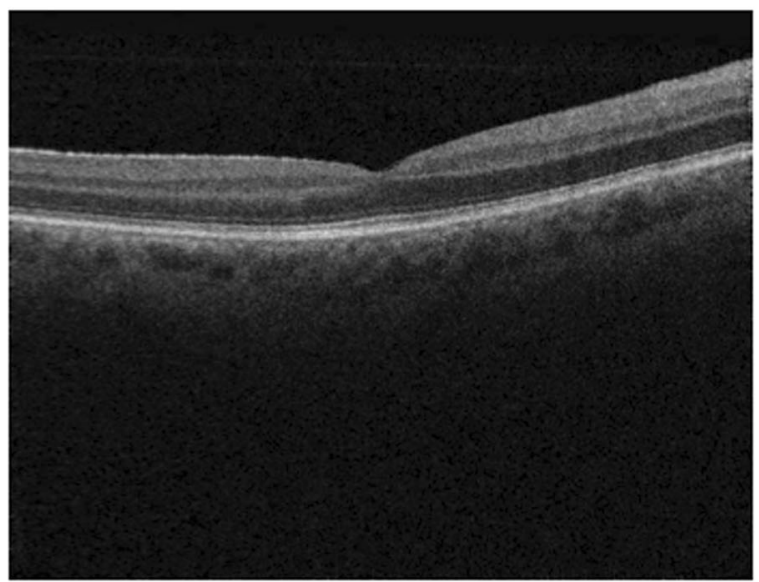

Fig. 1 a OCT Macula image of patient $A$ at 2 months following removal of HSO. b OCT Macula image of patient B at 2 months following removal of mixed HSO

were also suggested as a causative factor but sampling of retro-oil fluid has failed to support this theory [5].

The true incidence of silicone oil-related visual loss may indeed be higher, as patients with macula off RDs would not easily be identified using this definition of change in visual acuity.

\section{Conclusion}

The incidence of silicone oil-related visual loss following ROHSO was $4.9 \%$. These are the first two reported cases of SO maculopathy following the removal of HSO and mixed HSO. Further study of this unexplained phenomenon is warranted.

\section{Compliance with ethical standards}

Conflict of interest The authors declare that they have no conflict of interest.

Publisher's note: Springer Nature remains neutral with regard to jurisdictional claims in published maps and institutional affiliations.

\section{References}

1. Moya R, Chandra A, Banerjee PJ, et al. The incidence of unexplained visual loss following removal of silicone oil. Eye. 2015;29:1477-82.

2. Roca JA, Lihteh W, Berrocal $M$, et al. Un-explained visual loss following silicone oil removal: results of the Pan American Collaborative Retina Study (PACORES). Int J Retina Vitreous. 2017;3:26

3. Shalchi Z, Mahroo OA, Shunmugam M, et al. Spectral domain optical coherence tomography findings in long-term silicone oil-related visual loss. Retina. 2015;35:555-63.

4. Dogramaci M, Williams K, Lee E, Williamson TH. Foveal light exposure is increased at the time of removal of silicone oil with the potential for phototoxicity. Graefes Arch Clin Exp Ophthalmol. 2013;251:35-9.

5. Scheerlinck LM, Kuiper JJ, Liem AT, et al. Electrolyte composition of retro-oil fluid and silicone oil-related visual loss. Acta Ophthalmol. 2016;94:449-53. 